\title{
Predefined-Time Modified Function Projective Synchronization for Multiscroll Chaotic Systems via Sliding Mode Control Technology
}

\author{
Qiaoping $L i \mathbb{D}^{1,2}$ and Chao Yue ${ }^{1}$ \\ ${ }^{1}$ School of Economics, Zhengzhou University of Aeronautics, Zhengzhou 450015, China \\ ${ }^{2}$ School of Mathematics and Statistics, Xidian University, Xi'an 710071, China \\ Correspondence should be addressed to Qiaoping Li; liqiaoping1981@126.com
}

Received 21 April 2020; Accepted 9 July 2020; Published 19 August 2020

Academic Editor: Carlos Aguilar-Ibanez

Copyright (C) 2020 Qiaoping Li and Chao Yue. This is an open access article distributed under the Creative Commons Attribution License, which permits unrestricted use, distribution, and reproduction in any medium, provided the original work is properly cited.

\begin{abstract}
In the context of chaotic secure communication and based on the sliding mode control technology, this article investigates the predefined-time modified function projective synchronization for the Colpitts oscillator multiscroll hyperchaotic systems. Firstly, a four-dimensional multiscroll hyperchaotic system is introduced and the predefined-time synchronization is defined. Subsequently, applying a novel predefined-time stability criterion, an integral terminal sliding mode surface is constructed for the synchronization error system to ensure that the sliding motion is stable within a predefined time; meanwhile, an approaching controller is designed to enable the error system to reach and remain on the sliding mode surface within another predefined time, so as to ensure the realization of the predefined-time synchronization. Finally, the simulation experiments are presented to verify the effectiveness of derived theoretical results.
\end{abstract}

\section{Introduction}

During the last decades, the chaotic system has attracted more and more attention due to its potential applications in secure communication [1-4]. Chaotic synchronization is the premise to realize chaotic secure communication, and whose essence is that the state of the response system can be consistent with that of the drive system according to a particular relationship. Therefore, the intensive study of the synchronization scheme and synchronization control technology of chaotic systems can provide a solid theoretical support for the development of chaotic secure communication $[5,6]$.

The security is an important indicator to measure the performance of the communication scheme. Since the security performance of a chaotic system increases with its complexity, the carrier system with stronger chaotic characteristics can effectively improve the reliability of chaotic secure communication. However, most chaotic synchronization studies focus on chaotic systems with single scroll or double scroll, such as Chua system, Lorenz system, Rössler system, and L $\ddot{u}$ system. Compared with the traditional chaotic systems, multiscroll chaotic systems exhibit more complex topological structure and more unpredictable dynamic behavior. Therefore, adopting the multiscroll chaotic system as the carrier system is more beneficial to improve the attack resistance of the communication scheme [7-10].

Synchronization time is another important indicator to measure the performance of the communication scheme since the encoded message cannot be recovered before the synchronization is established. To realize fast synchronization, the finite-time control technique has been formulated. Compared with asymptotic control, finite-time control has many advanced properties, such as fast and finite-time convergence, high tracking accuracy, and good robustness to uncertainty. Therefore, finite-time synchronization has become more and more attractive in recent years [11-13].

However, the convergence time of finite-time stability depends heavily on the initial state of the system. In 
particular, when the initial state is far from the equilibrium point, the convergence time may be unbounded. To solve this problem, the fixed-time stability has been proposed, in which the convergence time is uniformly bounded for all initial states of the system; that is to say, the upper bound of the convergence time does not depend on the initial state of the system. The fixed-time stability has been applied to design robust control schemes for synchronizing chaotic systems [14-16]. However, even though the fixed-time stability has a significant advantage over the classical finitetime stability in some sense, the convergence (settling) time in fixed-time control cannot be preassigned because the relationship between the tuning gains and the upper bound of the settling time is unclear in general.

Additionally, in the process of secure communication, the designer expects the encoded message can be recovered within any sufficiently short time given in advance. It is necessary to consider the concept of predefined-time stability control technique, applying which an upper bound (sometimes the least upper bound) of convergence (settling) time is directly involved in the design of the controller as a preassigned tuning gain [17-20]. In chaotic secure communication, if the synchronization scheme is designed based on the above control technique, the encrypted message will be decrypted within a predetermined time which is independent of the initial state. This motivates our work.

Sliding mode control (SMC) is well known for its robustness to system parameter variations and external disturbances [21]. However, the traditional SMC schemes based on linear sliding surface can only guarantee the asymptotic convergence but not the finite-time convergence. In order to obtain better control performance, the terminal sliding mode control (TSMC) has been proposed by adding a nonlinear item into the sliding mode surface function, which can realize the finite-time stability of the system [22]. Nevertheless, there is serious chattering in both SMC and TSMC, which limits their practical application. To solve this problem, the integral terminal sliding mode control (ITSMC) is designed by introducing the integral compensation term into the function of terminal sliding mode surface, which can further weaken the chattering caused by the sign function $[23,24]$. It is a pity that most existing integral terminal sliding mode controls are single-power and lack of flexibility.

Moreover, as chaotic synchronization is applied in secure communication, the level of the communication security is dependent on the complexity level of the synchronization scheme. So far, many complex synchronization schemes have been proposed, among which the modified function projective synchronization (MFPS) is more general and it covers almost all of the existing synchronization methods [25-27]. Applying MFPS, the drive system and the response system are synchronized up to a desired scaling function matrix. To the best of our knowledge, the predefined-time modified function projective synchronization (MFPS) for chaotic systems is still open, which is another motivation of our work.

Motivated by the above discussion, in this article, the problem of predefined-time modified function projective synchronization is discussed for chaotic systems with multiple scrolls. Compared with the existing literature, the main contributions that make this work more competitive are summarized as below.

First, by introducing the predefined-time synchronization, the synchronization time can be preassigned according to the task requirements and it can be arbitrarily short. Second, adopting sliding mode control technology to realize the predefined-time stability not only can enhance the robustness of the system to external interference and uncertain parameters but also can determine the time of the sliding mode reaching phase and the settling time during the sliding mode phase by distributing the weights of time distribution, which is more flexible. Last but not the least, the multiscroll chaotic system applied in this work is convenient for physical realization, and the number of scrolls can be adjusted according to the designer's requirement, which is also flexible.

The rest of this article is organized as follows. In Section 2 , the multiscroll chaotic system and the synchronization problem are introduced. In Section 3, an novel terminal sliding mode control scheme is designed to realize the predefined-time synchronization. In Section 4, the secure communication experiment is carried out to prove the validity and the advancement of the proposed synchronization control scheme. Finally, the conclusion and future work are presented in Section 5.

\section{Preliminaries and Problem Description}

In this section, we will list some relevant preliminaries and introduce the synchronization problem.

2.1. Four-Dimensional Multiscroll Systems. Recently, Bao constructed a new four-dimensional multiscroll hyperchaotic system by modifying the original equation of the third-dimensional single-scroll chaotic Colpitts oscillator model with two piecewise linear triangular wave functions [10]. Its dynamical model is given as

$$
\left\{\begin{array}{l}
\dot{x}_{1}=\mu\left[x_{3}-g_{M}\left(x_{2}\right)\right], \\
\dot{x}_{2}=\mu\left[x_{3}+g_{N}\left(x_{4}\right)\right], \\
\dot{x}_{3}=-\frac{0.5\left(x_{1}+x_{2}\right)}{\mu}-x_{3}, \\
\dot{x}_{4}=x_{1}+x_{2},
\end{array}\right.
$$

in which

$$
\begin{aligned}
g_{K}(\xi)= & \sum_{k=-K, k \neq 0}^{K} \frac{p}{2 q}\left\{\left|\xi-p\left(2 k-\frac{|k|}{k}\right)+q\right|-\mid \xi\right. \\
& \left.-p\left(2 k-\frac{|k|}{k}\right)-q \mid\right\}-\xi,
\end{aligned}
$$


represents a piecewise linear triangular wave function. When we choose $\mu=1, p=1, q=0.02, M=2, N=1$ and $\left(x_{1}(0), x_{2}(0), x_{3}(0), x_{4}(0)\right)=(1,2,-1,-3)$, the chaotic attractor of system (1) and the function curves of $g_{M}\left(x_{2}\right)$ and $g_{N}\left(x_{4}\right)$ are shown in Figures 1-3 respectively.

Remark 1. When the parameter $\mu>0.6263$, the chaotic or hyperchaotic state of system (1) is robust and stable, and the number of chaotic scrolls in its chaotic attractor is determined by the parameters $M$ and $N$. The number of chaotic scrolls on phase planes $x_{1} \times x_{4}$ and $x_{2} \times x_{4}$ is $(2 M+1)(2 N+1)$, the number of chaotic scrolls on phase plane $x_{3} \times x_{4}$ is $2 N+1$, and the number of chaotic scrolls on the other three phase planes is $2 M+1$. Therefore, the number of chaotic scrolls can be changed by adjusting the values of the parameters $M$ and $N$, which has important application value in chaotic secure communication. For instance, if we reset $M=4, N=4$, then the chaotic attractor of system (1) is shown in Figure 4.

Remark 2. Since the state of chaotic system (1) is bounded, combined with the property of triangular wave function (2), one can derive that the composite functions $g_{M}\left(x_{2}\right)$ and $g_{N}\left(x_{4}\right)$ are also bounded, which can be shown in Figures 2-3.

Remark 3. Compared with several chaotic systems commonly used in chaotic secure communication which are shown in Figure 5, it is obvious that the multiscroll chaotic oscillator presented in this work is more complex, so it has better security.

\subsection{Predefined-Time Stability and Predefined-Time} Synchronization. Consider the dynamical system:

$$
\dot{v}(t)=\phi(t, v ; \sigma), \quad t \in[0,+\infty),
$$

where $v \in R^{n}$ stands for the system state and $\sigma \in R^{m}$ represents the constant parameter vector. Assume the origin is an equilibrium point of system (3), and denote $v_{0}=v(0)$; then, $\phi\left(0, v_{0} ; \sigma\right)=0$.

Definition 1 (finite-time stability) [13]. The origin of system (3) is said to be globally finite-time stable if it is globally asymptotically stable and any solution $v\left(t, v_{0}\right)$ of (3) reaches the equilibrium point within some finite time, i.e.,

$$
\begin{aligned}
\lim _{t \rightarrow T\left(v_{0}\right)_{-}} v\left(t, v_{0}\right) & =0, \\
v\left(t, v_{0}\right) & \equiv 0, \quad \forall t \geq T\left(v_{0}\right),
\end{aligned}
$$

in which $T\left(v_{0}\right)=\inf \left\{\varepsilon \geq 0: \lim _{t \longrightarrow \varepsilon} v\left(t, v_{0}\right)=0\right\}$ is called the settling-time function of system (3).

Definition 2 (fixed-time stability) [16]. The origin of system (3) is said to be fixed-time stable if it is globally finite-time stable and the settling-time function $T\left(v_{0}\right)$ is bounded, i.e., there exists a positive constant $T_{\max }$ such that $T\left(v_{0}\right) \leq T_{\max }, \forall v_{0} \in R^{n}$.

Definition 3 (predefined-time stability) [19]. The origin of system (3) is said to be globally predefined-time stable if it is fixed-time stable and the settling-time function $T\left(v_{0}\right)$ satisfies

$$
T\left(v_{0}\right) \leq T_{c}, \quad \forall v_{0} \in R^{n},
$$

in which $T_{c}$ is a finite positive constant given in advance, and we called it the predefined time.

Lemma 1 (see [20]). Let $T_{c}>0$ be a predefined constant. If there exists a radially unbounded Lyapunov function $V(\nu)$ for dynamic system (3) and it satisfies

$$
\begin{aligned}
& \dot{V}(\nu)=g(V(\nu)), \\
& g(V) \leq-\frac{\pi}{\alpha T_{c}}\left(V^{1+(\alpha / 2)}+V^{1-(\alpha / 2)}\right),
\end{aligned}
$$

where $\alpha \in(0,1)$ is a real constant.

Then, the origin of system (3) is globally predefined-time stable with a predefined time $T_{c}$.

Proof. For any $v_{0} \in R^{n}$, the settling-time function $T\left(v_{0}\right)$ complies to

$$
\begin{aligned}
T\left(v_{0}\right) & =\int_{0}^{T\left(v_{0}\right)} \mathrm{d} t=\int_{V\left(v_{0}\right)}^{0} \frac{1}{g(V)} \mathrm{d} V \\
& \leq \int_{V\left(v_{0}\right)}^{0}-\frac{1}{\left(\pi / \alpha T_{c}\right)\left(V^{1+(\alpha / 2)}+V^{1-(\alpha / 2)}\right)} \mathrm{d} V \\
& =\frac{\alpha T_{c}}{\pi} \int_{0}^{V\left(v_{0}\right)} \frac{\mathrm{d} V}{V^{1+(\alpha / 2)}+V^{1-(\alpha / 2)}} \\
& =\frac{\alpha T_{c}}{\pi} \int_{0}^{V\left(v_{0}\right)} \frac{\mathrm{d} V}{V^{1-(\alpha / 2)}\left(1+V^{\alpha}\right)} \\
& =\frac{\alpha T_{c}}{\pi} \int_{0}^{V\left(v_{0}\right)} \frac{(2 / \alpha) \mathrm{d} V^{(\alpha / 2)}}{1+V^{\alpha}} \\
& =\left.\frac{\alpha T_{c}}{\pi} \cdot \frac{2}{\alpha} \arctan \left(V^{(\alpha / 2)}\right)\right|_{0} ^{V\left(v_{0}\right)} \\
& =\frac{2 T_{c}}{\pi} \arctan \left(V\left(v_{0}\right)^{(\alpha / 2)}\right) .
\end{aligned}
$$

Since $\quad V\left(v_{0}\right) \in[0,+\infty)$, which implies $\arctan \left(V\left(v_{0}\right)^{(\alpha / 2)}\right) \in(0,(\pi / 2)]$. Hence, we obtain $T\left(v_{0}\right) \leq T_{c}$ and

$$
T_{c}=\sup T\left(v_{0}\right)
$$

Definition 4 (modified function projective synchronization) [25]. Two chaotic dynamical systems

$$
\begin{aligned}
& \dot{x}(t)=h_{1}(t, x), \\
& \dot{y}(t)=h_{2}(t, y)
\end{aligned}
$$

are said to be modified function projective synchronization (MFPS), if there exists a reversible scaling matrix $\Lambda(t)$ such that 

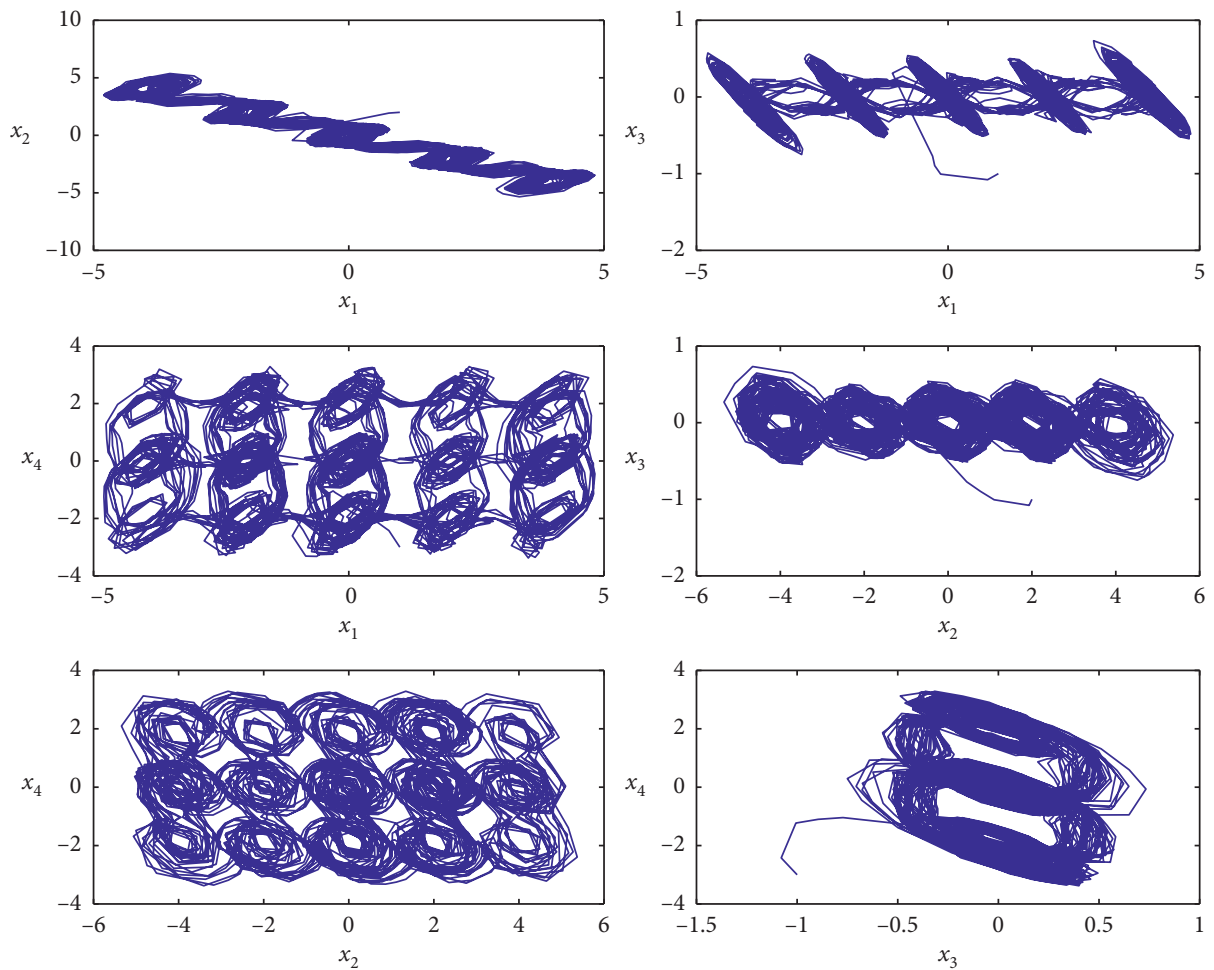

FIgURE 1: Multiscroll chaotic attractor of system (1) with $M=2$ and $N=1$.

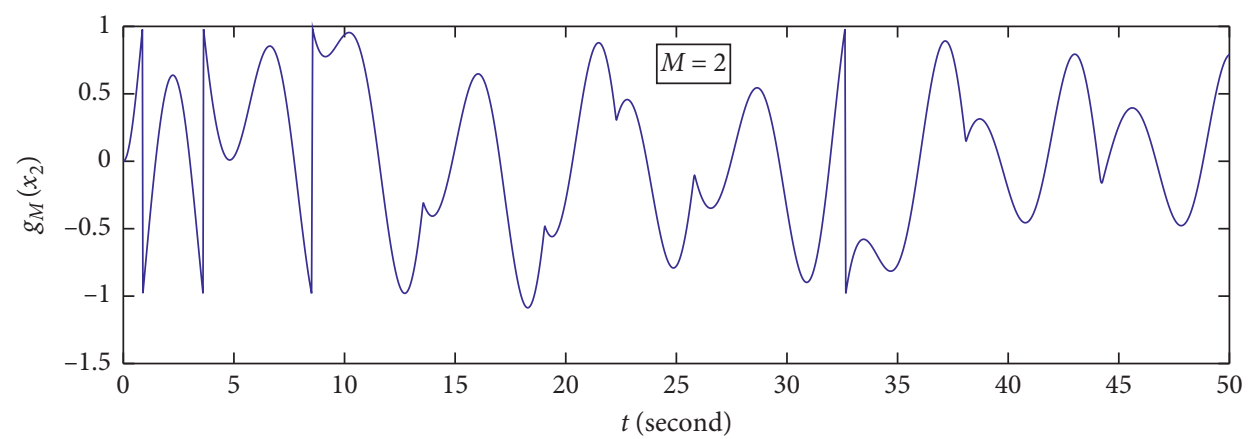

Figure 2: The function curve of $g_{M}\left(x_{2}\right)$ in system (1) with $M=2$ and $N=1$.

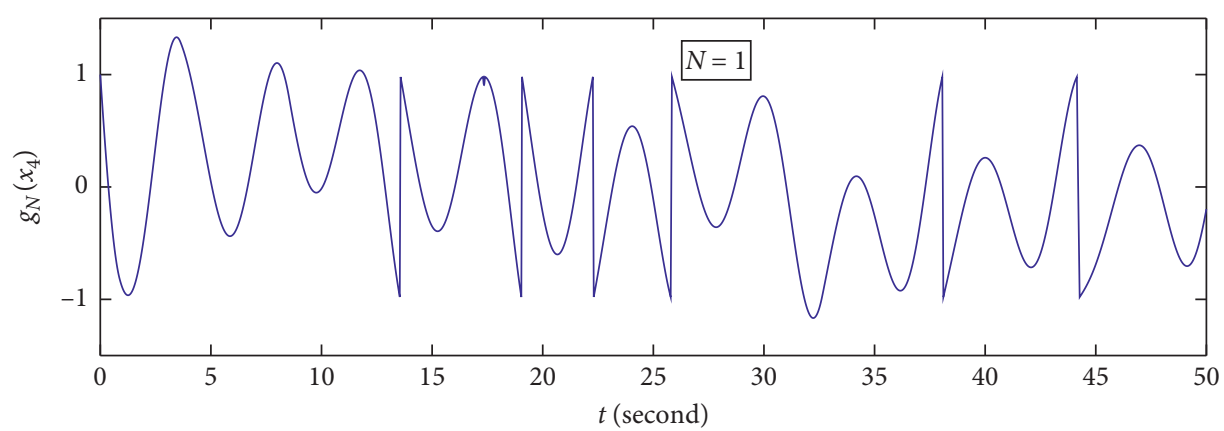

Figure 3: The function curve of $g_{N}\left(x_{4}\right)$ in system (1) with $M=2$ and $N=1$.

$$
\lim _{t \rightarrow \infty}\|y(t)-\Lambda(t) x(t)\|=0,
$$

where $x(t), y(t) \in R^{n}$ stand for the state vectors for the above two chaotic systems, respectively,
$\Lambda(t)=\operatorname{diag}\left\{\lambda_{1}(t), \ldots, \lambda_{n}(t)\right\}$, and $\lambda_{i}(t)$ is a bounded and continuously differentiable nonzero function, $i=1,2, \ldots, n$.

Denote $e(t)=y(t)-\Lambda(t) x(t)$, and we can obtain the error dynamical system between (9) and (10): 

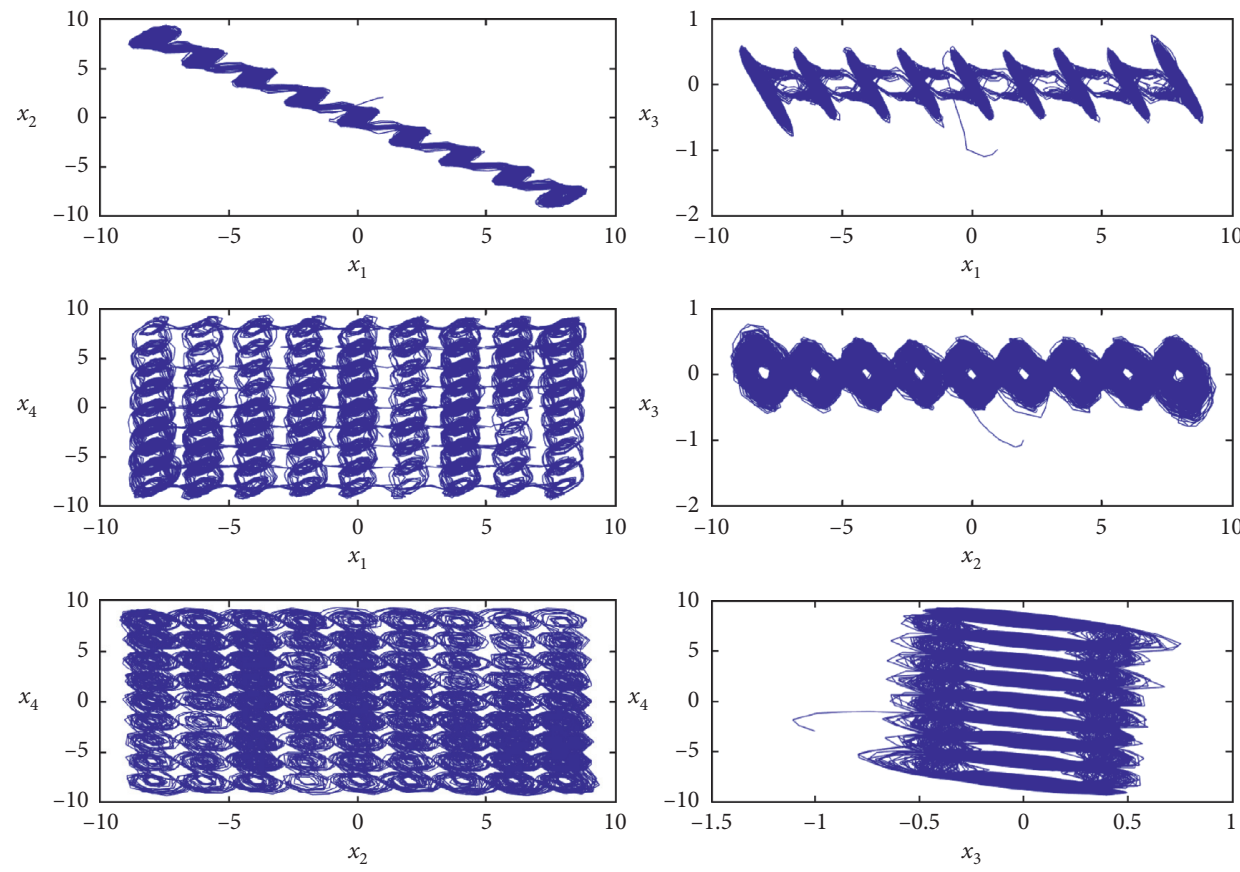

FIgURE 4: Multiscroll chaotic attractor of system (1) with $M=4$ and $N=4$.
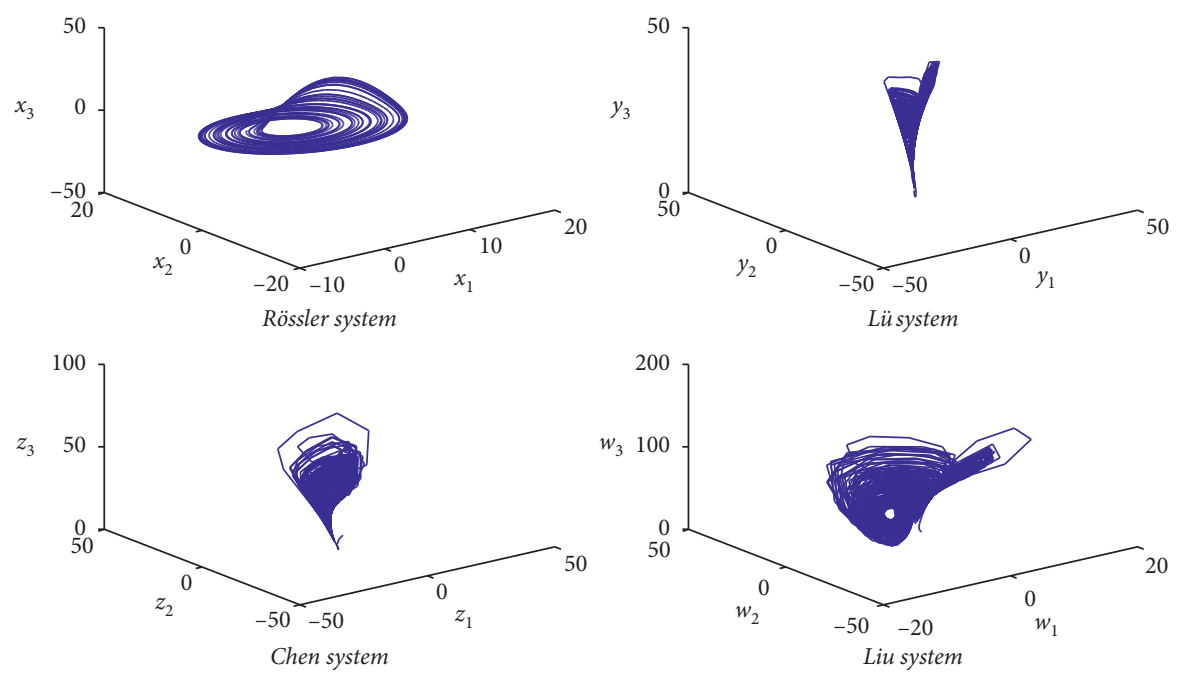

FIGURE 5: Chaotic attractor of several chaotic systems commonly used in chaotic secure communication.

$$
\dot{e}(t)=h_{2}(t, y)-\Lambda(t) h_{1}(t, x)-\dot{\Lambda}(t) h_{1}(t, x)
$$

Definition 5. It is said that the two chaotic systems mentioned in Definition 1 are predefined-time modified function projective synchronization if and only if the origin of error dynamical system (12) is globally predefined-time stable with predefined time $T_{c}>0$.

$$
\begin{aligned}
A & =\left(\begin{array}{rrrr}
0 & 0 & \mu & 0 \\
0 & 0 & \mu & 0 \\
-\frac{0.5}{\mu} & -\frac{0.5}{\mu} & -1 & 0 \\
1 & 1 & 0 & 0
\end{array}\right), \\
f(x) & =\left[f_{1}(x), f_{2}(x), f_{3}(x), f_{4}(x)\right]^{T} \\
& \triangleq\left[-\mu g_{M}\left(x_{2}\right), \mu g_{N}\left(x_{4}\right), 0,0\right]^{T},
\end{aligned}
$$


and then system (1) can be rewritten as

$$
\begin{aligned}
\dot{x}(t) & =A x(t)+f(t), \\
\text { or } \dot{x}_{i}(t) & =A_{i} x(t)+f_{i}(x), \quad i=1, \cdots, 4 .
\end{aligned}
$$

In this work, multiscroll chaotic system (14) is adopted as the drive system, and the response system is chosen as

$$
\dot{y}(t)=A y(t)+f(y)+u(t)
$$

where $u(t) \in R^{4}$ is the vector of control input.

The main objective in this work is to design a sliding mode control scheme to achieve the predefined-time modified function projective synchronization of (14) and (16). Since the predefined-time synchronization problem is equivalent to the predefined-time stabilization problem of the error system, now let us define the MFPLS error vector:

$$
e(t)=y(t)-\Lambda(t) x(t)
$$

from which the corresponding error dynamic system is obtained as

$$
\begin{aligned}
& \dot{e}_{i}(t)=\dot{y}_{i}(t)-\lambda_{i}(t) \dot{x}_{i}(t)-\dot{\lambda}_{i}(t) x_{i}(t) \\
&=A_{i} e(t)+f_{i}(y)-\lambda_{i}(t) f_{i}(x)-\dot{\lambda}_{i}(t) x_{i}(t)+u_{i}(t), \\
& i=1,2,3,4 .
\end{aligned}
$$

Let $\overline{f_{i}}(x, y)=f_{i}(y)-\lambda_{i}(t) f_{i}(x)$, and error system (18) is simplified into

$$
\begin{array}{r}
\dot{e}_{i}(t)=A_{i} e(t)+\overline{f_{i}}(x, y)-\dot{\lambda}_{i}(t) x_{i}(t)+u_{i}(t), \\
i=1,2,3,4 .
\end{array}
$$

Combining the boundedness of $\lambda_{i}(t)$ and Remark 2, we can obtain that the function $\overline{f_{i}}(x, y)$ is also bounded. In this work, we assume that there exists known nonnegative constant $d_{i}$ such that

$$
\left\|\overline{f_{i}}(x, y)\right\| \leq d_{i}, \quad i=1,2,3,4 .
$$

\section{Design of the Predefined-Time Control Scheme}

In this section, we will focus on the design of predefinedtime synchronization control scheme by means of terminal sliding mode control technology. This control scheme will be realized by two steps: (1) construct a nonsingular terminal sliding surface to ensure the predefined-time stable of the sliding motion. (2) Design an approaching control law to guarantee the occurrence of the sliding motion in predefined time.

3.1. Sliding Mode Phase. In this work, a novel nonsingular integral terminal sliding mode switching function is introduced as follows:

$$
\begin{aligned}
S_{i}(t)= & e_{i}(t)+\int_{0}^{t}\left(c_{i 1} e_{i}(\sigma)+c_{i 2} \operatorname{sgn}\left(e_{i}(\sigma)\right)\left|e_{i}(\sigma)\right|^{1+\alpha_{1}}\right. \\
& \left.+c_{i 3} \operatorname{sgn}\left(e_{i}(\sigma)\right)\left|e_{i}(\sigma)\right|^{1-\alpha_{1}}\right) \mathrm{d} \sigma,
\end{aligned}
$$

where $0<\alpha_{1}<1, \quad c_{i 1}>0, \quad c_{i 2}=\left(\pi / 2^{1+\left(\alpha_{1} / 2\right)} \alpha_{1} T_{1, c}\right), \quad$ and $c_{i 3}=\left(\pi / 2^{1-\left(\alpha_{1} / 2\right)} \alpha_{1} T_{1, c}\right)$ represent the sliding mode parameters, $i=1,2,3,4$.

Remark 4. Compared with the following two traditional integral terminal sliding mode switching functions proposed in $[24,28]$,

$$
S_{i}(t)=e_{i}(t)+c \int_{0}^{t} \operatorname{sgn}\left(e_{i}(\sigma)\right)\left|e_{i}(\sigma)\right|^{\alpha} \mathrm{d} \sigma, \quad c>0,0<\alpha<1,
$$

$S_{i}(t)=e_{i}(t)+c \int_{0}^{t} e_{i}(\sigma) \mathrm{d} \sigma, \quad c>0$,

sliding mode switching function (21) has the following advantage: the factor $c_{i 1} e_{i}+c_{i 2} \operatorname{sgn}\left(e_{i}\right)\left|e_{i}\right|^{2-\alpha_{1}}$ plays the leading role in guaranteeing a fast convergence speed as $\left|e_{i}(t)\right|$ is much larger than 1 , while the factor $c_{i 3} \operatorname{sgn}\left(e_{i}\right)\left|e_{i}\right|^{\alpha_{1}}$ is the dominant one when $\left|e_{i}(t)\right|$ is much less than 1 , so as to ensure the fast convergence speed throughout the sliding mode stage.

Let

$$
S_{i}(t)=\dot{S}_{i}(t)=0, \quad i=1,2,3,4,
$$

and the dynamics of sliding mode can be obtained as follows:

$$
\begin{aligned}
\dot{e}_{i}(t)= & -\left(c_{i 1} e_{i}(t)+c_{i 2} \operatorname{sgn}\left(e_{i}(t)\right)\left|e_{i}(t)\right|^{1+\alpha_{1}}\right. \\
& \left.+c_{i 3} \operatorname{sgn}\left(e_{i}(t)\right)\left|e_{i}(t)\right|^{1-\alpha_{1}}\right), \quad i=1,2,3,4 .
\end{aligned}
$$

Theorem 1. For a predefined time $T_{c}>0$, sliding mode (25) is predefined-time stable and the trajectory $e(t)$ converges to the equilibrium $e(t)=0$ within time $T_{1, c}=\beta_{1} T_{c}$ and the constant gain $\beta_{1} \in(0,1)$.

Proof. Construct the following Lyapunov function:

$$
V_{1, i}(t)=\frac{1}{2} e_{i}^{2}(t), \quad i=1,2,3,4
$$

By taking the derivative of $V_{1, i}(t)$ along the trajectory of system (17), we obtain

$$
\begin{aligned}
\dot{V}_{1, i}(t) & =e_{i}(t) \dot{e}_{i}(t) \\
& =-\left(c_{i 1}\left(e_{i}(t)\right)^{2}+c_{i 2}\left|e_{i}(t)\right|^{2+\alpha_{1}}+c_{i 3}\left|e_{i}(t)\right|^{2-\alpha_{1}}\right) \\
& =-2 c_{i 1} V_{1, i}-\frac{\pi}{\alpha_{1} T_{1, c}}\left(\left(V_{1, i}\right)^{1+\left(\alpha_{1} / 2\right)}+\left(V_{1, i}\right)^{1-\left(\alpha_{1} / 2\right)}\right) \\
& \leq-\frac{\pi}{\alpha_{1} T_{1, c}}\left(\left(V_{1, i}\right)^{1+\left(\alpha_{1} / 2\right)}+\left(V_{1, i}\right)^{1-\left(\alpha_{1} / 2\right)}\right) .
\end{aligned}
$$


Applying Lemma 1, we can directly deduce that each element $e_{i}(t)$ converges to zero in the predefined time $T_{1, c}$ during the sliding mode phase, where $i=1,2,3,4$. This yields the error vector $e(t)$ converges to $e(t)=0$ within $T_{1, c}$.

Remark 5. As shown in Figure 6, compared with (22) and (23), sliding mode switching function (21) designed in this work can ensure error system (19) converges to the equilibrium point $e_{i}(t)=0$ faster during the sliding mode phase, and the convergence time can be preassigned, so it is more flexible. Here, the error system is started from $e_{i}(0)=0$ with $i=1$, and the convergence time during the sliding mode phase based on (21) is preappointed as $T_{1, c}=0.1$.

3.2. Sliding Mode Approaching Phase. Until now, the sliding surface has been established. In order to ensure the existence of the sliding mode in the predefined time, the following sliding mode approaching control law is designed:

$$
\begin{aligned}
u_{i}(t)= & -\left[c_{i 1} e_{i}+c_{i 2} \operatorname{sgn}\left(e_{i}\right)\left|e_{i}\right|^{1+\alpha_{1}}+c_{i 3} \operatorname{sgn}\left(e_{i}\right)\left|e_{i}\right|^{1-\alpha_{1}}\right. \\
& \left.+k_{1} \operatorname{sgn}\left(S_{i}\right)\left|S_{i}\right|^{1+\alpha_{2}}+k_{2} \operatorname{sgn}\left(S_{i}\right)\left|S_{i}\right|^{1-\alpha_{2}}\right] \\
& -d_{i} \operatorname{sgn}\left(S_{i}\right)-A_{i} e+\dot{\lambda}_{i}(t) x_{i}(t)
\end{aligned}
$$

where $k_{1}=\left(\pi / 2^{1+\left(\alpha_{2} / 2\right)} \alpha_{2} T_{2, c}\right)$ and $k_{2}=\left(\pi / 2^{1-\left(\alpha_{2} / 2\right)} \alpha_{2} T_{2, c}\right)$ are two control gains, and $i=1,2,3,4$.

Theorem 2. For the predefined time $T_{c}$ given in Theorem 1, applying control law (28), the error trajectory $e_{i}(t)$ of (19) will reach the sliding surface $S_{i}(t)=0$ within the predefined time $T_{2, c}=\beta_{2} T_{c}$ and remain on it forever. Here, the weighted gain $\beta_{2}$ satisfies $\beta_{2} \in(0,1)$ and $\beta_{1}+\beta_{2}=1$.

Proof. Selecting the following Lyapunov function, we get

$$
V_{2, i}(t)=\frac{1}{2} S_{i}^{2}(t), \quad i=1,2,3,4 .
$$

Taking the time derivative of $V_{2, i}(t)$, we get

$$
\begin{aligned}
\dot{V}_{2, i}(t)= & S_{i}(t) \dot{S}_{i}(t) \\
= & S_{i}\left[\dot{e}_{i}+\left(c_{i 1} e_{i}+c_{i 2} \operatorname{sgn}\left(e_{i}\right)\left|e_{i}\right|^{1+\alpha_{1}}+c_{i 3} \operatorname{sgn}\left(e_{i}\right)\left|e_{i}\right|^{1-\alpha_{1}}\right)\right] \\
= & S_{i}\left(\bar{f}_{i}(x, y)-d_{i} \operatorname{sgn}\left(S_{i}\right)\right)-S_{i} k_{1} \operatorname{sgn}\left(S_{i}\right)\left|S_{i}\right|^{1+\alpha_{2}} \\
& -S_{i} k_{2} \operatorname{sgn}\left(S_{i}\right)\left|S_{i}\right|^{1-\alpha_{2}} \\
\leq & \left(d_{i}\left|S_{i}\right|-d_{i}\left|S_{i}\right|\right)-k_{1}\left|S_{i}\right|^{2+\alpha_{2}}-k_{2}\left|S_{i}\right|^{2-\alpha_{2}} \\
= & -k_{1}\left|S_{i}\right|^{2+\alpha_{2}}-k_{2}\left|S_{i}\right|^{2-\alpha_{2}} \\
= & -\frac{\pi}{\alpha_{2} T_{2, c}}\left(\left(V_{2, i}\right)^{1+\left(\alpha_{2} / 2\right)}+\left(V_{2, i}\right)^{1-\left(\alpha_{2} / 2\right)}\right) .
\end{aligned}
$$

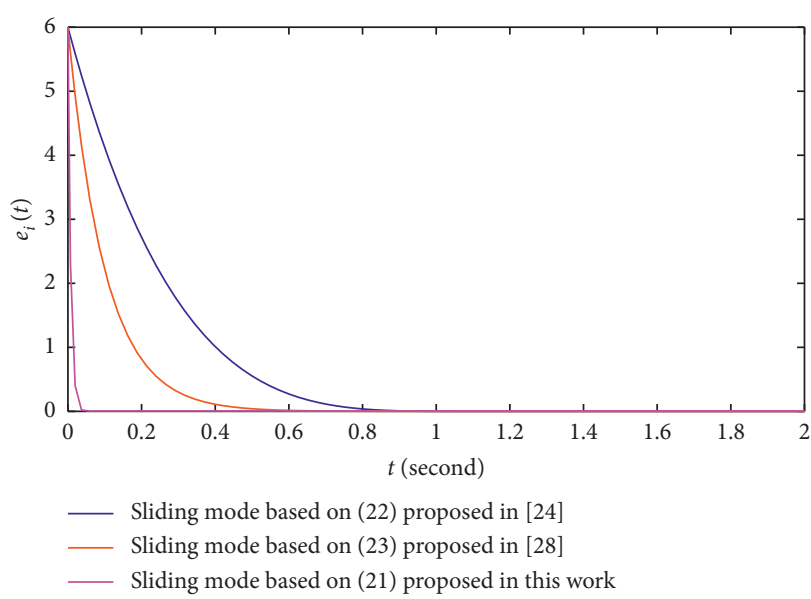

FIGURE 6: Comparison of the three sliding modes mentioned in this work with $e_{i}(0)=6$.

Hence, from Lemma 1, it follows that the error trajectory $e_{i}(t)$ can reach the sliding surface $S_{i}(t)=0$ in the predefined time $T_{2, c}$ and then remain on it forever.

Remark 6. Combining the results of Theorems 1 and 2, applying control law (28), systems (14) and (16) can realize modified function projective synchronization within the predefined time $T_{1, c}+T_{2, c}=\beta_{1} T_{c}+\beta_{2} T_{c}=T_{c}$. The designer can determine the time length of the sliding mode reaching phase and the stable settling-time length during the sliding mode phase by distributing the time distribution weight according to the task requirement.

\section{Simulation Experiment}

In this section, we will present a chaotic secure communication simulation to verify and illustrate the effectiveness of the proposed predefined-time synchronization scheme.

The framework of chaotic secure communication is shown in Figure 7, and the basic principle is as follows.

At the transmitting end, a chaotic signal $x(t)$ is generated by the drive system as the carrier wave signal, and then the signal $s(t)$ transmitted by the sender is superimposed with the carrier signal $x(t)$ and modulated into a hybrid signal such as $w(t)=K_{s} x(t)+s(t)$, which will be transmitted to the receiver through the transmission channel. Meanwhile, at the receiver end, the response system tracks the state of the drive system under the action of the synchronous controller $u(t)$ and reverses the carrier signal $\widehat{x}(t)$, and then it recovers the original signal $s(t)=w(t)-$ $K_{s} \widehat{x}(t)$ with its received signal $w(t)$ to realize chaotic secure communication. In the process of chaotic secure communication, there are two kinds of signals transmitted in the signal transmission channel, one is the modulated signal $w(t)$ and the other is $y(t)$. Even if the attacker intercepts the above two signals, if he wants to obtain the original information $s(t)$, he needs to get the secret keys including the chaotic synchronization scheme and the signal modulation scheme. Therefore, increasing the complexity of the 


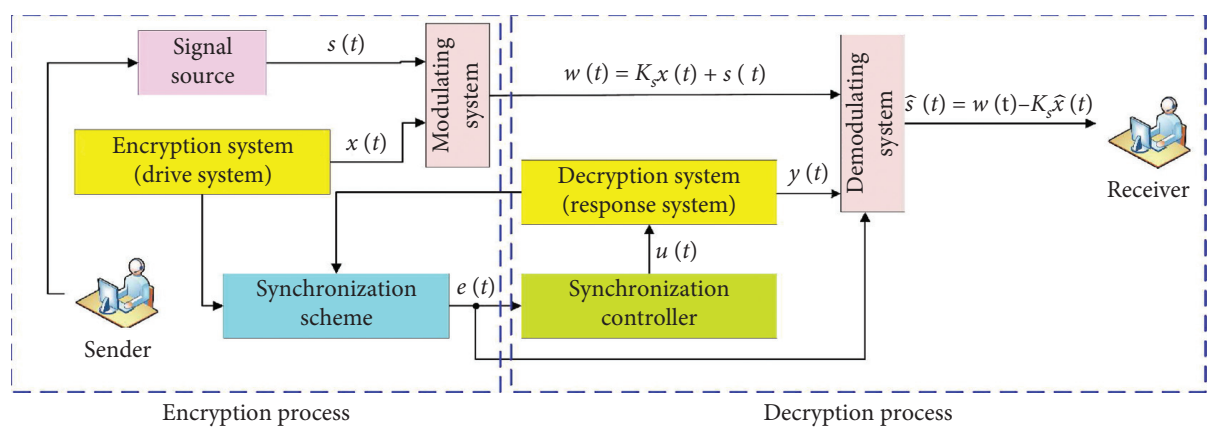

FIgURE 7: Framework of the chaotic secure communication.

synchronization scheme and signal modulation scheme is the main way to improve the security of secure communication. Meanwhile, the more chaotic and complex the carrier signal is, the better its concealment is. Therefore, improving the complexity of the carrier signal is another effective mean to improve the antiaggression of the chaotic modulation scheme.

In this simulation, the four-dimensional multiscroll hyperchaotic systems (14) and (16) introduced in Section 1 are considered as the encryption system (the drive system) and the decryption system (the response system), respectively. The system parameters are taken as $\mu=1, p=1, q=0.02, M=2$, and $N=1$, and the initial state value are randomly chosen as $x(0)=(1,2,-1,-3)$ and $y(0)=(5,1,-1,-1)^{T}$.

Based on Definition 5, the synchronization scheme is designed as

$$
\begin{aligned}
\lim _{t \longrightarrow T_{c^{-}}}\|e(t)\| & =\lim _{t \longrightarrow T_{c^{-}}}\|y(t)-\Lambda(t) x(t)\|=0, \\
\|e(t)\| & =\|y(t)-\Lambda(t) x(t)\| \equiv 0, \\
& \text { if } t \geq T_{c},
\end{aligned}
$$

in which $T_{c}>0$ is the predefined synchronization time, and the scaling matrix is given as

$$
\begin{aligned}
\Lambda(t)= & \operatorname{diag}\left\{\lambda_{1}(t), \lambda_{2}(t), \lambda_{3}(t), \lambda_{4}(t)\right\}=\operatorname{diag}\{1+0.2 \sin t,-1 \\
& +0.5 \cos t, 3-0.5 \sin t,-5-0.4 \cos 2 t\},
\end{aligned}
$$

and the upper bound vector of $\left\|\bar{f}_{i}(x, y)\right\|$ is given by $\left(d_{1}, d_{2}, d_{3}, d_{4}\right)=(4,4,0,0)$.

Meanwhile, in the simulation experiment of secure communication, the encrypted signal (the original signal) is given as

$$
s(t)=0.8 \sin 2 t
$$

In order to enhance the concealment of the communication scheme, in the signal modulation process, it is usually required to modulate the original signal in the carrier signal at a relatively small ratio which is less than $10 \%$. According to the above principles, the modulation scheme is designed as

$$
w(t)=s(t)+K_{s} x(t)=s(t)+2 x_{1}(t)-x_{2}(t)+6 x_{3}(t)-2.5 x_{4}(t) .
$$

From the comparison of Figure 8 , one can see that, based on modulation scheme (34), the bandwidth of the encrypted signal $s(t)$ is less than that of the carrier signal $K_{s} x(t)$, and the modulated signal $w(t)$ is more complex and more difficult to predict than $s(t)$, which indicates that the modulated scheme can well hide the original signal.

Based on Theorems 1 and 2, we carry out the simulation with predefined-time sliding mode control scheme (21) and (28) proposed in Section 3. The parameters in the switching function of the sliding mode surface are taken as $\alpha_{1}=0.25, \alpha_{2}=0.2$, and $c_{i 1}=10$, the predefined synchronization time is given as $T_{c}=0.2$, and the weighted gains in sliding mode stage and sliding mode approaching stage are allotted as $\beta_{1}=0.5$ and $\beta_{2}=0.5$, respectively. Furthermore, the parameters dependent on $T_{c}$ can be obtained by calculating $c_{i 2}=\left(\pi / 2^{1+\left(\alpha_{1} / 2\right)} \alpha_{1} \beta_{1} T_{c}\right)$, $c_{i 3}=\left(\pi / 2^{1-\left(\alpha_{1} / 2\right)} \alpha_{1} \beta_{1} T_{c}\right), \quad k_{1}=\left(\pi / 2^{1+\left(\alpha_{1} / 2\right)} \alpha_{2} \beta_{2} T_{c}\right), \quad$ and $k_{2}=\left(\pi / 2^{1-\left(\alpha_{1} / 2\right)} \alpha_{2} \beta_{2} T_{c}\right)$. Then, the simulation results are shown in Figures 9-11.

As shown in Figures 9-10, even if the synchronization time $T_{c}$ is set to be very small, the synchronization scheme proposed in this work can still ensure that the switching function of the sliding mode surface $S_{i}(t)$ reaches and remains on the sliding mode surface $S_{i}(t)=0$ within the predefined time $T_{2, c}=\beta_{2} T_{c}=0.1$ and the MFPS error $e_{i}(t)=0$ converges to zero within the predefined time $T_{c}=0.2 ;$ thus, the predefined-time MFPS of systems (14) and (16) is realized successfully.

According to the chaotic secure communication scheme shown in Figure 7, during the decryption process, the receiver can use the secret keys to decrypt the original signal as follows:

$$
\begin{aligned}
\widehat{s}(t)= & w(t)-\left[2\left(\lambda_{1}(t)\right)^{-1} y_{1}(t)-\left(\lambda_{2}(t)\right)^{-1} y_{2}(t)\right. \\
& \left.+6\left(\lambda_{3}(t)\right)^{-1} y_{3}(t)-2.5\left(\lambda_{4}(t)\right)^{-1} y_{4}(t)\right] .
\end{aligned}
$$

The state trajectories of the encrypted signal $s(t)$ and the decrypted signal $\widehat{s}(t)$ are shown in Figure 11, from which one can see that the decrypted signal can track the original signal quickly in the desired time $T_{c}=0.2$ and keep consistent with it, which shows the effectiveness of the proposed secure communication scheme. 


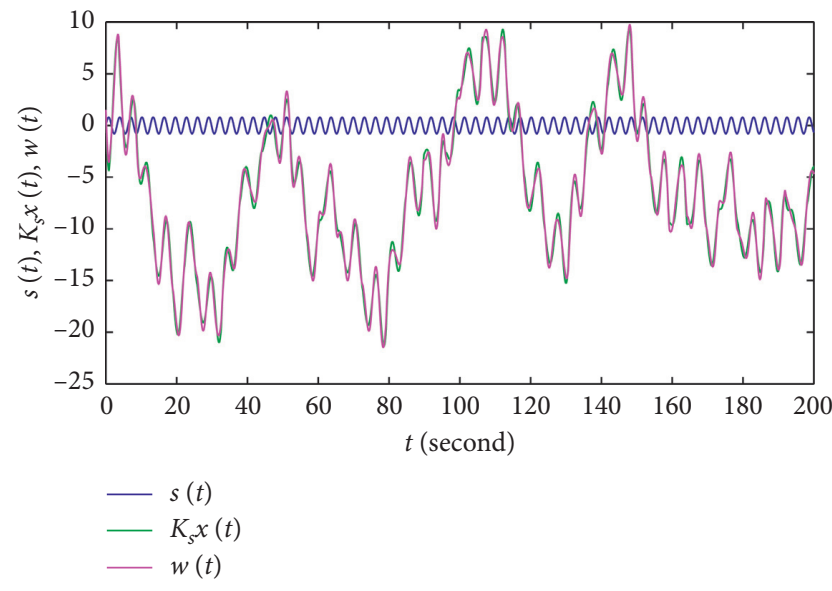

FIGURE 8: Comparison among the encrypted signal $s(t)$, the carrier signal $K_{s} x(t)$, and the modulated signal $w(t)$.

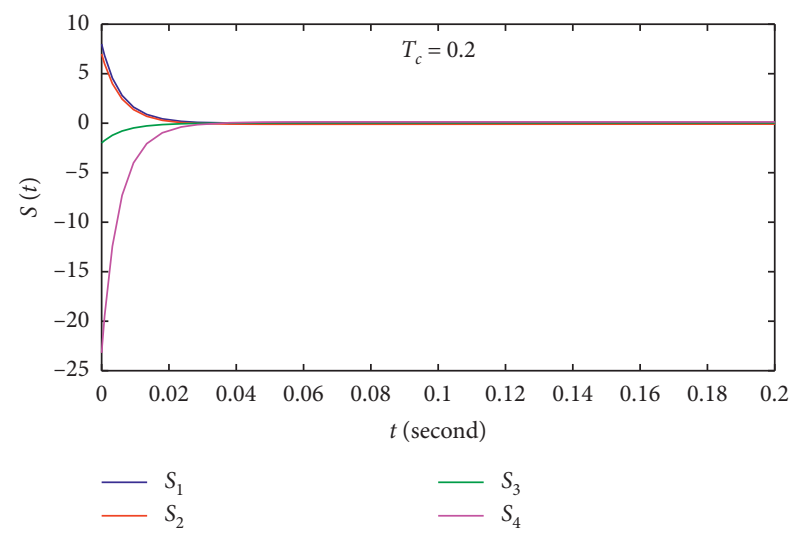

Figure 9: Time response of sliding mode surface switching function $S(t)$ with $T_{c}=0.2$.

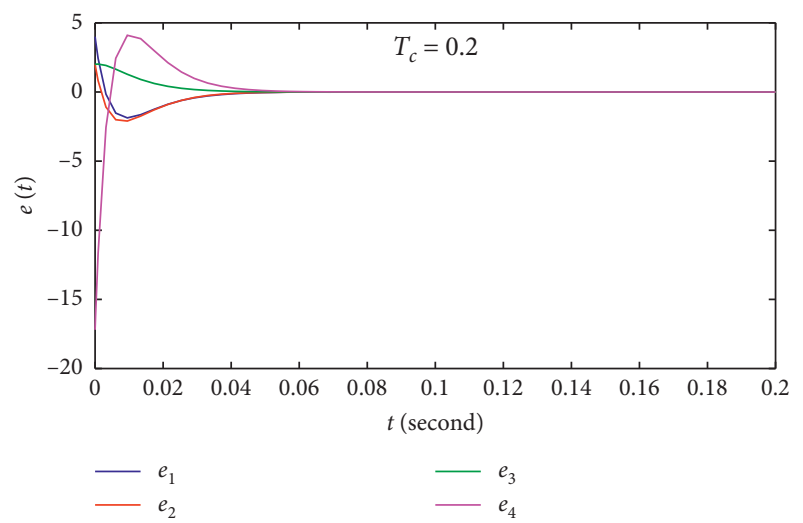

FIgURE 10: Time response of MFPS error $e$ with $T_{c}=0.2$.

To further verify the advancement of the synchronization scheme designed in this work, we shorten the predefined synchronization time to $T_{c}=0.02$. From the simulation results shown in Figures 12-14, one can see that

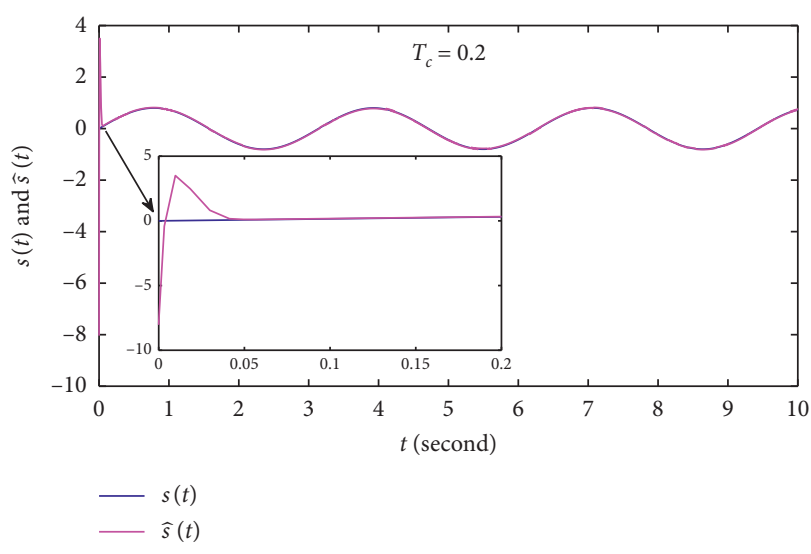

FIGURE 11: State trajectories of encrypted signal $s(t)$ and decrypted signal $\widehat{s}(t)$ with $T_{c}=0.2$.

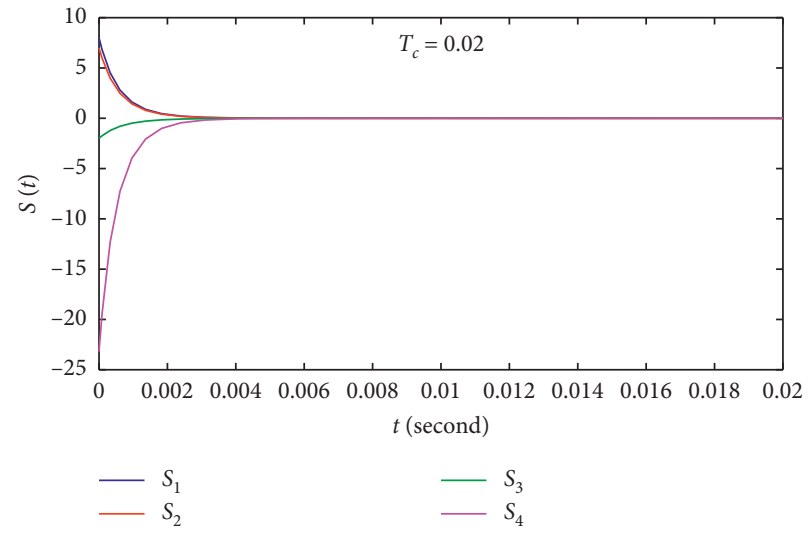

FIGURE 12: Time response of sliding mode surface switching function $S(t)$ with $T_{c}=0.02$.

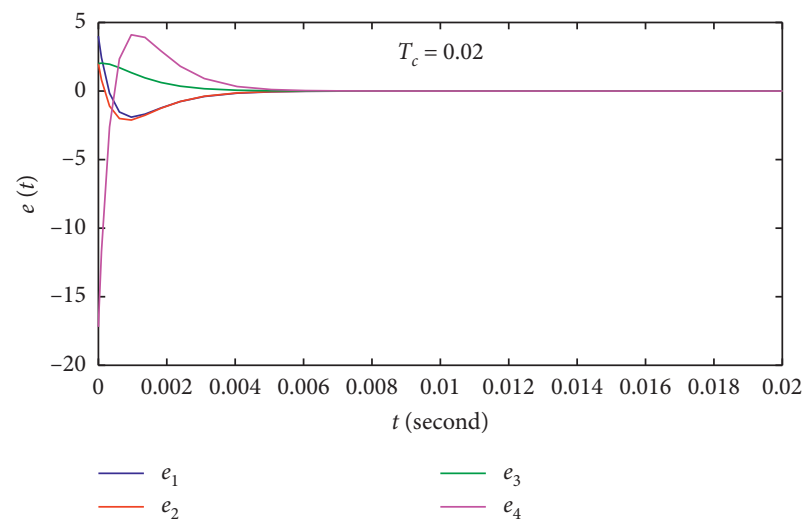

FIgURE 13: Time response of MFPS error $e(t)$ with $T_{c}=0.02$.

the synchronization control scheme proposed in this work can still ensure that the encoded message can be recovered within the predefined time $T_{c}=0.02$, which indicates the high efficiency of the proposed predefined-time control scheme. 


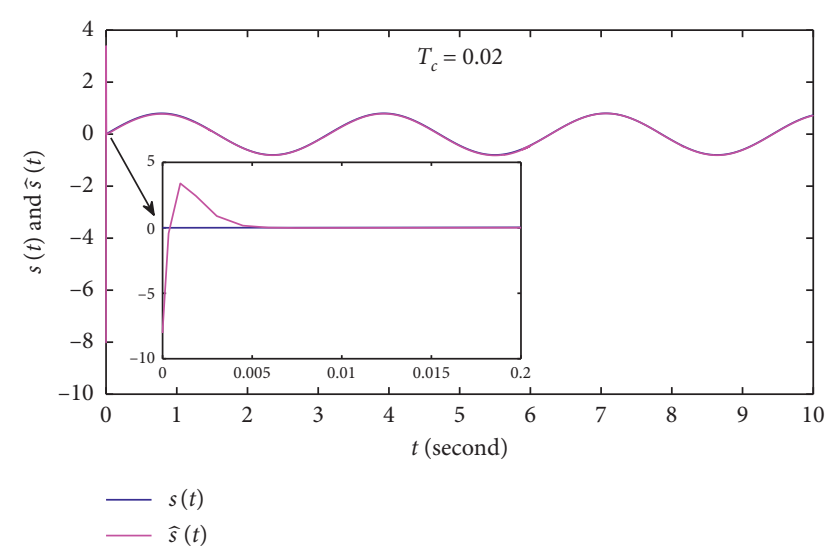

FIGURE 14: State trajectories of encrypted signal $s(t)$ and decrypted signal $\widehat{s}(t)$ with $T_{c}=0.02$.

\section{Conclusion}

In this article, we dealt with the problem of the predefinedtime modified function projective synchronization (MFPS) for a class of multiscroll chaotic systems. Based on Lyapunov stability theory, a novel predefined-time stability criterion was proposed, applying which a terminal sliding mode control scheme was designed to realize the modified function projective synchronization within a predefined time. Finally, the simulation of secure communication showed that the proposed synchronization control scheme can ensure that the encoded message can be recovered within arbitrarily short time given in advance. The sliding mode control used in this work is well known for its robustness to system parameter variations and external disturbances. Moreover, applying the proposed synchronization control scheme, the designer can determine the time length of the sliding mode reaching phase $T_{2, c}$ and the stable settling-time length during the sliding mode phase $T_{1, c}$ by adjusting the time distribution weight $\beta_{1}$ and $\beta_{2}$ according to the task requirement. The smaller the parameter $\beta_{2}$, the shorter the sliding mode approaching time and the better the system robustness. This means that the control scheme designed in this work can effectively deal with the relatively weak noise to ensure the good communication quality. It is worth emphasizing that, when the noise is strong, if the noise is strong, it is necessary to design a filter, noise observer, or an adaptive controller for the proposed synchronous control scheme. This topic is valuable and challenging, and we will study it in detail in the follow-up work. In addition, the consensus of multiagent systems can be regarded as the generalization of synchronization problem and our further work is to deal with the predefined-time consensus of multiagent systems applying the terminal sliding mode control scheme designed in this work, which is also a meaningful and challenging subject.

\section{Data Availability}

The data used to support the findings of this study are included within the article.

\section{Conflicts of Interest}

The authors declare that there are no conflicts of interest regarding the publication of this article.

\section{Acknowledgments}

This work was supported by the National Natural Science Foundation of China (grant numbers: 11702201 and 61877046) and the Key Scientific Research Projects of Higher Education Institutions in Henan Province (grant numbers: 19B110006 and 20A110034).

\section{References}

[1] L. Ma, Z. Wang, and H.-K. Lam, "Event-triggered meansquare consensus control for time-varying stochastic multiagent system with sensor saturations," IEEE Transactions on Automatic Control, vol. 62, no. 7, pp. 3524-3531, 2017.

[2] X. Chai, J. Zhang, Z. Gan, and Z. Yushu, "Medical image encryption algorithm based on Latin square and memristive chaotic system," Multimedia Tools and Applications, vol. 78, pp. $35419-435453,2019$.

[3] J.-J. He and B.-C. Lai, "A novel 4 d chaotic system with multistability: dynamical analysis, circuit implementation, control design," Modern Physics Letters B, vol. 33, no. 21, Article ID 1950240, 2019.

[4] S. Zhang, Y. Zeng, Z. Li, M. Wang, and L. Xiong, "Generating one to four-wing hidden attractors in a novel $4 \mathrm{~d}$ no-equilibrium chaotic system with extreme multistability," Chaos, vol. 28, no. 1, Article ID 013113, 2018.

[5] U. E. Kocamaz, S. Çiçek, and Y. Uyaroğlu, "Secure communication with chaos and electronic circuit design using passivity-based synchronization," Journal of Circuits, Systems and Computers, vol. 27, no. 4, Article ID 1850057, 2018.

[6] C. Nwachioma, J. H. Perez-Cruz, A. Jimenez, M. Ezuma, and R. Rivera-Blas, "A new chaotic oscillator-properties, analog implementation, and secure communication application," IEEE Access, vol. 7, pp. 7510-7521, 2019.

[7] J. M. Muñoz-Pacheco, E. Zambrano-Serrano, O. FélixBeltrán, L. C. Gómez-Pavón, and A. Luis-Ramos, "Synchronization of pwl function-based $2 \mathrm{~d}$ and $3 \mathrm{~d}$ multi-scroll chaotic systems," Nonlinear Dynamics, vol. 70, no. 2, pp. 1633-1643, 2012.

[8] M. A. Khelifa and A. Boukabou, "Design of an intelligent prediction-based neural network controller for multi-scroll chaotic systems," Applied Intelligence, vol. 45, no. 3, pp. 793-807, 2016.

[9] W. M. Ahmad, "Generation and control of multi-scroll chaotic attractors in fractional order systems," Chaos, Solitons \& Fractals, vol. 25, no. 3, pp. 727-735, 2005.

[10] B. Bao, Z. Liu, J. Xu, and L. Zhu, "Generation of multi-scroll hyperchaotic attractor based on colpitts oscillator model," ACTA Physica Sinica, vol. 59, no. 3, pp. 1540-1548, 2010.

[11] Y. Li, X. Yang, and L. Shi, "Finite-time synchronization for competitive neural networks with mixed delays and nonidentical perturbations," Neurocomputing, vol. 185, no. 10, pp. 242-253, 2016.

[12] X. Liu, H. Su, and M. Z. Q. Chen, “A switching approach to designing finite-time synchronization controllers of coupled neural networks," IEEE Transactions on Neural Networks and Learning Systems, vol. 27, no. 2, pp. 471-482, 2016. 
[13] S. P. Bhat and D. S. Bernstein, "Finite-time stability of continuous autonomous systems," SIAM Journal on Control and Optimization, vol. 38, no. 3, pp. 751-766, 2000.

[14] G. Ji, C. Hu, J. Yu, and H. Jiang, "Finite-time and fixed-time synchronization of discontinuous complex networks: a unified control framework design," Journal of the Franklin Institute, vol. 355, no. 11, pp. 4665-4685, 2018.

[15] X. Yang, J. Lam, D. W. C. Ho, and Z. Feng, "Fixed-time synchronization of complex networks with impulsive effects via nonchattering control," IEEE Transactions on Automatic Control, vol. 62, no. 11, pp. 5511-5521, 2017.

[16] A. Polyakov, "Nonlinear feedback design for fixed-time stabilization of linear control systems," IEEE Transactions on Automatic Control, vol. 57, no. 8, pp. 2106-2110, 2012.

[17] A. J. M. noz Vázquez, J. D. S. Torres, and C. A. A. Gij'n, "Single-channel predefined-time synchronisation of chaotic systems," Asian Journal of Control, vol. 1, no. 2, pp. 1-9, 2019.

[18] E. Jiménez-Rodríguez, J. D. Sânchez-Torres, and A. G. Loukianov, "On optimal predefined-time stabilization," in Proceedings of the XVII Latin American Conference of Automatic Control, pp. 317-322, Medellin, Colombia, October, 2016.

[19] J. D. Sánchez-Torres, D. Gmez-Gutiérrez, E. López, and A. G. Loukianov, "A class of predefined-time stable dynamical systems," IMA International Journal of Robust and Nonlinear Control, vol. 35, no. 1, pp. 1-23, 2018.

[20] A. A.-G. Carlos, J. M. n.-V. Aldo, D. S.-T. Juan, R.-G. Gerardo, and M.-R. Fernando, "On predefined-time synchronisation of chaotic systems," Chaos, Solitons and Fractals, vol. 122, pp. 172-178, 2019.

[21] C. R. Bheesayagari, J. Pons-Nin, M. T. Atienza, and M. Dominguez-Pumar, "Diffusive representation and sliding mode control of charge trapping in Al\$_2\$O\$3 MOS capacitors," IEEE Transactions on Industrial Electronics, vol. 66, no. 11, pp. 8628-8637, 2019.

[22] H. Du, X. Chen, G. Wen, X. Yu, and J. Lü, “Discrete-time fast terminal sliding mode control for permanent magnet linear motor," IEEE Transactions on Industrial Electronics, vol. 65, no. 12, pp. 9916-9927, 2018.

[23] C.-S. Chiu, "Derivative and integral terminal sliding mode control for a class of mimo nonlinear systems," Automatica, vol. 48, no. 2, pp. 316-326, 2012.

[24] M. P. Aghababa, S. Khanmohammadi, and G. Alizadeh, "Finite-time synchronization of two different chaotic systems with unknown parameters via sliding mode technique," Applied Mathematical Modelling, vol. 35, no. 6, pp. 30803091, 2011.

[25] H. Du, Q. Zeng, and C. Wang, "Modified function projective synchronization of chaotic system," Chaos, Solitons \& Fractals, vol. 42, no. 4, pp. 2399-2404, 2009.

[26] $\mathrm{D}$. Li, "Modified functional projective synchronization of the unidirectional and bidirectional hybrid connective star network with coupling time-delay," Wuhan University Journal of Natural Sciences, vol. 24, no. 4, pp. 321-328, 2019.

[27] R. Kumar and S. Das, "Weak, modified and function projective synchronization of cohencgrossberg neural networks with mixed time-varying delays and parameter mismatch via matrix measure approach," Neural Computing and Applications, vol. 32, no. 11, pp. 1-12, 2019.

[28] M. Hou and Y. Wang, "A model-free adaptive integral terminal sliding mode control method," Control and Decision, vol. 33, no. 9, pp. 1591-1597, 2018. 